\title{
Analysis and Design of University Open Laboratory Management System
}

\author{
Xie Hongyan \\ Comprehensive Practice Center of Economic and Management, School of Management, Harbin University of \\ Commerce, Harbin, 150028, Heilongjiang, China \\ xhy0710@163.com
}

Keywords: open experimental teaching; laboratory management system; system analysis and design

\begin{abstract}
Open experimental teaching is the direction of experimental teaching reform in colleges and universities. The networked and open experimental teaching and laboratory management information platform established by using network technology can make the laboratory open management more information-based and scientific. In view of the common problems existing in the laboratories of colleges and universities at present, and combining with the actual requirements, this paper carries out a comprehensive design from the aspects of system architecture, functional modules, database and security. The design scheme will help improve the management level of university laboratories.
\end{abstract}

\section{Introduction}

Laboratory is an important part of colleges and universities, and is one of the basic conditions for running a good college. At present, the construction of open laboratory has become a hot spot in university laboratory work, which has brought greater challenges to experimental teaching managers. [1]Therefore, in the open network environment, it is urgent to develop a system that can meet the requirements of open laboratory management.

\section{Common Problems in Laboratory Management Systems}

With the continuous expansion of experimental facilities and the continuous increase of experimental courses, higher requirements have been put forward for the management of laboratory information system. Problems commonly existing in laboratory management systems at present:

(1) Lack of comprehensive management of laboratory management personnel, technology, environment and the use of experimental equipment, and lack of evaluation mechanism for the investment benefit of experimental construction;

(2) In terms of the information management in the laboratory, Excel tables are mainly used as the tools to add, update, store, query and statistic data. The data operation is prone to errors, resulting in difficulties in later management, query and statistics [2].

(3) The information cannot be shared through the network. Teachers and students do not know about the use of laboratory and experimental equipment, and the experimental requirements and operating procedures of each laboratory;

(4) Lack of communication between lab managers, between administrators and teachers and students, and between laboratories and departments at all levels;

(5) Cannot generate various statistics and reports according to various query conditions.

To solve these problems, it is necessary to analyze, integrate and reconstruct existing systems and management methods, and develop an open laboratory management information system that is more flexible and meets various requirements. 


\section{Requirements Analysis of Open Laboratory Management System}

\subsection{Announcement Information}

Publish the information of experiment teaching, let the user know the latest news and use situation related to experiment teaching. The published function is only available to the administrator, who can modify and delete messages. The average user can read the message[3].

\subsection{System User Login}

Users can only use the system functionality after logging in. The system has three types of users: student users, lab users and system administrator users. The permission of the student user is to make an appointment for experiment, inquire the selected experiment, view the experimental project information, query the current course arrangement and course selection distribution, query the score, leave a message to the teacher, and modify personal information. The permission of the laboratory personnel is to inquire and print the reservation list, inquire the specific course selection, enter students' experimental results, inquire students' information, leave messages to students and modify personal information; System administrator for system management and maintenance.

\subsection{Information Management of Experimental Projects}

The experiment that the student can reserve must be the experiment project that the laboratory personnel has already carried out the open setting. The setting of open experimental project includes the experimental project, open period, course information, teacher information, etc.

\subsection{Experiment Appointment}

The student appointment experiment is the core function of the system. The information of the experimental project that the students choose to reserve includes the name of the laboratory, the name of the experimental project, date, time period, laboratory location, etc. [4]

\subsection{Query Reservation Experiment}

At any time, the student can inquire the information of the experiment he has booked. You can either query the individual items of the experimental project or query all the reserved content.

\section{Design of Open Laboratory Management System}

\subsection{System Architecture}

Layer 1: the front-end client browser. The browser makes an application to the WEB server using the HTTP protocol, and the WEB server processes the client's request and returns the result to the client browser in HTML format. [5]

Layer 2: middleware Web servers. Consisting of Windows 2010, iis7.0 and.net operating platforms and management systems, the school network Web server is selected for actual operation. The Web server applies ASP. NET technology to realize dynamic data invocation on the server side, forming dynamic interactive pages, and using Javascript and.net controls to realize dynamic application pages on the client side, playing the role of information transmission relay.

Layer 3: background database server. Select SQL Server 2010, the system adopts B/S three-layer architecture, and USES ADO. NET objects provided by ASP. NET to access the database.

\subsection{Functional Module Design}

It consists of two major parts: the client side and the management side. The client is composed of the student user module and the teacher user module. The student user module provides various functions for students to select courses online. [6]The teacher user module provides all the functions of teaching management for students in the course of experimental teaching. 
The management side has the functions of searching, adding, deleting and modifying the experimental project, student list, experimental course arrangement, student's course selection record, etc. It is composed of five functional modules, namely, academic management, teaching management, experimental appointment, performance management and system management. It also has three levels of management authority, and different levels of administrators have different functions. The system functional framework is shown in figure 1.

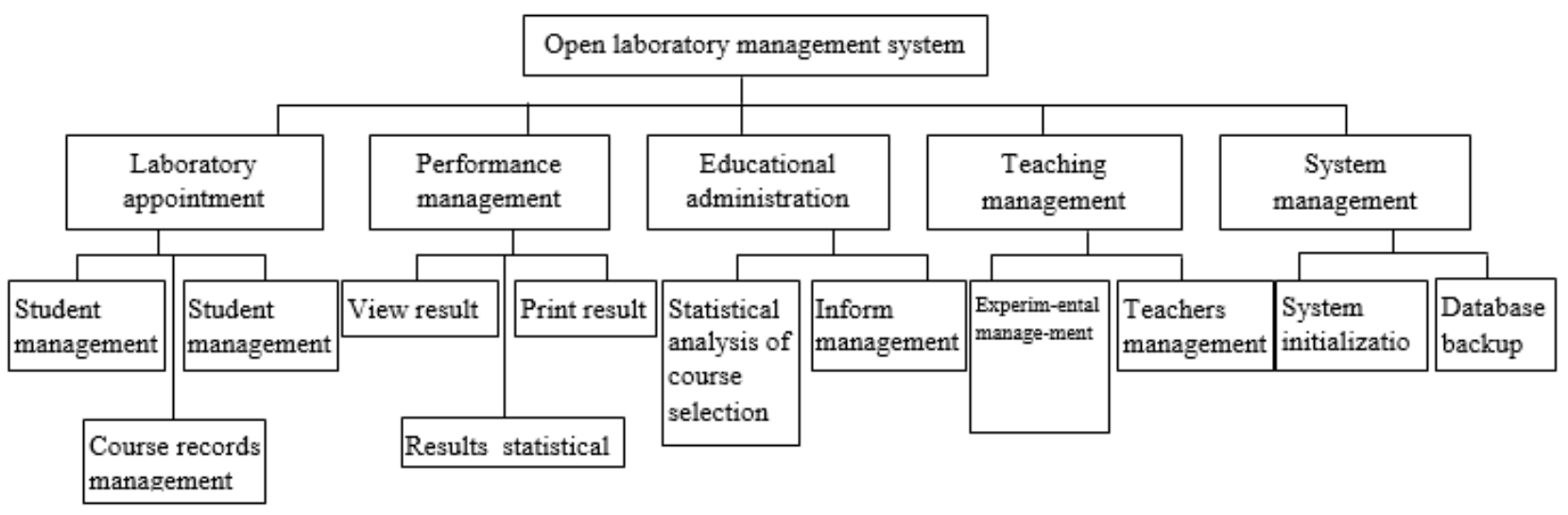

Fig. 1 System function diagram

\subsection{System Database Design}

In the database design of this system, a total of 7 tables are designed according to the system requirements and database design theory. The main tables and table items are as follows:

(1) Manager information table: mainly includes user name, password and authorization.

(2) Student information form: mainly includes student number, class, name, login password, course selection, E-mail, telephone, remarks.

(3) Course information table: it mainly includes course name, starting department number, course description and remarks.

(4) Laboratory building information table: it mainly includes laboratory building name, house number and number of people.

(5) Information table of experimental classrooms: it mainly includes the name of the laboratory, house number and the number of people who can be accommodated.

(6) Information table of experimental teachers: it mainly includes the name, work number and courses taught.

(7) Information table of course selection for students: it mainly includes course selection time, teacher number, course number, experiment number, experiment project number and student number.

\subsection{Database Connections and Access}

Since each module of the system uses the database, the connection and access to the database must be handled. [7] The system uses a multi-tier architectural model, so the database connection string needs to be passed between the Web services layer calling the business logic layer and the data access layer. The connection of this string can be changed, and it cannot be fixed at any level in the system, otherwise the code will have to be changed later when the server changes. So you first need to configure these string parameters in the web.config file, and when you change the web service later, maintenance will be very convenient. Therefore, the idea of database connection is to define the following string in web.config:

$<$ add key $=$ " str DBServer" value $=$ " . " / >
$<$ add key $=$ " str DBUser" value $=$ " sa" / >
$<$ add key = " str DBPwd" value = "123! @ \#" / > 


\subsection{Design for Safety}

(1) The user password is encrypted and stored in the database, and the user password is forced to use strong password to ensure that the password provided by the user meets certain complexity requirements.

(2) For user input, verify the type, length, format and scope of input on the client side to check the correct data. In addition, verify user input on the server side to prevent malicious design attacks on the system.

(3) In order to prevent hackers from gaining control of the data, it belongs to the SQL Server stored procedure and does not use the "inline query". This can greatly reduce the number of attempts to insert additional SQL commands into the input stream. Each stored procedure can also set storage permissions separately to improve security.

(4) window-based verification and role-based verification are adopted. [8]The Web site is partitioned, the public access part and the restricted part that requires authentication, the role of verified users is different, and the available resources are different, such as some pages that teachers can access that students cannot access.

\section{Summary}

The development of computer technology and information technology provides a new model for open laboratory management: open laboratory management model based on network environment. At the same time, the construction of campus network provides strong support for realizing this model. The open laboratory management system established in the network environment completely replaces the traditional artificial management mode, effectively improves the management level of experimental teaching, reflects the prominent position of experimental teaching in the quality education, and meets the requirements of modern teaching and management.

\section{Acknowledgements}

This work was financially supported by practice teaching reform and research project of Harbin University of commerce (SJXM2017B003) and national undergraduate innovation and entrepreneurship training program (201710240071).

\section{References}

[1] He jin zhejiang, Xu jing. Exploration and Research on Resource Sharing Mechanism of University Laboratories [J]. Laboratory Science, 2015, 13 (6): 132-135.

[2] $\mathrm{Wu}$ Mei. Preliminary Study on Constructing Education Resource Sharing Mechanism in Beijing-tianjin-hebei Region [J]. Tianjin Economy, 2014,198 (11): 14-18.

[3] Gao Tiegang, Zhang jia, Ma jian. Basic Research on Education Information Teaching Resource Sharing Model [J]. Education, 2012(6): 70-74.

[4] Xin Chunhong. Study on SQL Server Database Security [J]. Information Technology, 2009(5): 174-176.

[5] Li Chunbao, Zhao bingxiu, Zhang mu, Database System Development Tutorial -- Based on SQLServer2005 + VB [M]. Beijing: tsinghua university press, 2008.

[6] Tang Chenghua. Research on the Safety of Teaching Management Information System in Universities [J]. Science and Technology Innovation Guide, 2009(26): 4-4.

[7] Liu shuyun. Design and Implementation of College Instrument and Equipment Management Information System [J]. Laboratory Research and Exploration, 2011, 30 (1): 174-177.

[8] Xiao pengbo, Lu yanqing. Design and Implementation of University Open Laboratory Management System [J]. Experimental Science and Technology, 2011, 9 (5): 57-59. 